\title{
R-matrix calculation of integral and differential cross sections for low-energy electron impact excitations of $\mathbf{N}_{2}$ molecule
}

\author{
Motomichi Tashird* and Keiji Morokuma \\ Department of Chemistry, Emory University, \\ 1515 Dickey Drive, Atlanta, Georgia 30322, USA.
}

(Dated: October 22, 2018)

\begin{abstract}
Low-energy electron impact excitations of $\mathrm{N}_{2}$ molecules are studied using the fixed-bond R-matrix method based on state-averaged complete active space SCF orbitals. Thirteen target electronic states of $\mathrm{N}_{2}$ are included in the model within a valence configuration interaction representations of the target states. Integrated as well as differential cross sections of the $A^{3} \Sigma_{u}^{+}, B^{3} \Pi_{g}, W^{3} \Delta_{u}, B^{\prime 3} \Sigma_{u}^{-}, a^{\prime 1} \Sigma_{u}^{-}, a^{1} \Pi_{g}, w^{1} \Delta_{u}$ and $C^{3} \Pi_{u}$ states are calculated and compared with the previous experimental measurements. These excitations, especially of the higher four states, have not been studied enough theoretically in the previous literature. In general, good agreements are observed both in the integrated and differential cross sections. However, some discrepancies are seen in the integrated cross sections of the $A^{3} \Sigma_{u}^{+}$and $C^{3} \Pi_{u}$ states, especially around a peak structure.

PACS numbers: $34.80 . G s$
\end{abstract}

*Present address: Fukui Institute for Fundamental Chemistry, Kyoto University, Takano-Nishi-Hiraki-cho 34-4, Kyoto 606-8103, JAPAN.; E-mail:tashiro@ fukui.kyoto-u.ac.jp 


\section{INTRODUCTION}

Electron impact excitation of nitrogen molecules plays an important role in atmospheric emission of planets and satellites such as the Earth, Titan and Triton. For example, excitation of the $a^{1} \Pi_{g}$ state and subsequent transitions to the ground $X^{1} \Sigma_{g}^{+}$state are responsible for the far ultraviolet emissions of the Lyman-Birge-Hopfield system which is prominent in the airglow of the Earth's atmosphere[1]. Recently, Khakoo et al.[2] measured differential cross sections (DCSs) of electron impact excitation of $\mathrm{N}_{2}$ molecule from the ground $X^{1} \Sigma_{g}^{+}$state to the 8 lowest excited electronic states of $A^{3} \Sigma_{u}^{+}, B^{3} \Pi_{g}, W^{3} \Delta_{u}, B^{\prime 3} \Sigma_{u}^{-}, a^{\prime 1} \Sigma_{u}^{-}, a^{1} \Pi_{g}, w^{1} \Delta_{u}$ and $C^{3} \Pi_{u}$ states. Based on their differential cross section data, Johnson et al.[3] derived integral cross sections (ICSs) for these electron impact excitations. In general, their ICSs are smaller than the other experimental cross sections at low impact energies below $30 \mathrm{eV}$. These deviations may have some significance on study of atmospheric emissions, because a mean kinetic energy of electron at high altitudes is about $10 \mathrm{eV}[4]$. To shed light on this situation from a theoretical point of view, we perform the ab initio R-matrix calculations of electron impact excitations of $\mathrm{N}_{2}$ molecule in this work.

Many previous experimental measurements have been focused on excitation to a specific electronic state. For example, Ajello and Shemansky[5] and Mason and Newell[6] measured ICSs for electron impact excitation to the $a^{1} \Pi_{g}$ state, whereas Poparic et al.[7], Zubek[8] and Zubek and King [9] measured cross sections for the $C^{3} \Pi_{u}$ state. In addition to these works, Zetner and Trajmar [10] reported excitation cross sections to the $A^{3} \Sigma_{u}^{+}, B^{3} \Pi_{g}, W^{3} \Delta_{u}$ and $a^{1} \Pi_{g}$ states. So far, comprehensive measurements of the excitation to the 8 lowest electronic states are limited to three groups of Cartwright et al. [11], Brunger and Teubner [12] and Khakoo et al.[2]. The measurements of Brunger and Teubner [12] include excitation DCSs for the $E^{3} \Sigma_{o}^{+}$and $a^{\prime \prime} \Sigma_{g}^{+}$states in addition to the 8 lowest excited states. The DCSs of Brunger and Teubner[12] and Khakoo et al.[2] were later converted to ICSs by Campbell et al.[13] and Johnson et al.[3], respectively. Detailed reviews on electron $\mathrm{N}_{2}$ collisions can be found in Itikawa[14] and Brunger and Buckman[15].

Several groups have performed theoretical calculation of low energy electron collisions with $\mathrm{N}_{2}$ molecule. For example, Chung and Lin[16] employed the Born approximation to calculate excitation cross sections for the 11 target states including the $A^{3} \Sigma_{u}^{+}, B^{3} \Pi_{g}, W^{3} \Delta_{u}, a^{1} \Pi_{g}, w^{1} \Delta_{u}$ and $C^{3} \Pi_{u}$ states. Later, the same group of Holley et al.[17] calculated excitation ICSs for the $a^{1} \Pi_{g}$ state using a two-state-close-coupling method. Fliflet et al.[18] and Mu-Tao and McKoy[19] reported distorted-wave cross sections for excitation of the $A^{3} \Sigma_{u}^{+}, B^{3} \Pi_{g}, W^{3} \Delta_{u}, w^{1} \Delta_{u}, C^{3} \Pi_{u}, E^{3} \Sigma_{g}^{+}, b^{\prime 1} \Sigma_{u}^{+}$ 
and $c^{\prime 1} \Sigma_{u}^{+}$states. In general, these approximate methods are expected to be accurate at high impact energies above $30 \mathrm{eV}$. However, more elaborate method is required for precise comparison with experiment at low energies. Gillan et al.[20] calculated excitation ICSs for the $A^{3} \Sigma_{u}^{+}, B^{3} \Pi_{g}$ and $W^{3} \Delta_{u}$ states using the fixed nuclei R-matrix method. They included the 4 lowest target states in their R-matrix model, with target CI wave functions containing 2-13 CSFs. Their cross sections for the $A^{3} \Sigma_{u}^{+}$and $W^{3} \Delta_{u}$ states agree well with the experimental results of Cartwright et al. [11]. However, ICSs for the $B^{3} \Pi_{g}$ state deviate considerably from the experimental cross sections. Subsequently, they extended their R-matrix model to include the 8 lowest valence states[21]. Their target $\mathrm{CI}$ wave functions were much improved from their previous work by employing valence active space description, resulting in 68-120 CSFs per target state. In their paper, the ICSs were shown for the $A^{3} \Sigma_{u}^{+}, B^{3} \Pi_{g}, W^{3} \Delta_{u}$ and $B^{\prime 3} \Sigma_{u}^{-}$states, while the DCSs were presented for only the $A^{3} \Sigma_{u}^{+}$state. Agreement with the ICSs of Cartwright et al.[11] is good for these 4 excited states. However, agreement is marginal at DCS level.

In this work, we study electron impact excitation of $\mathrm{N}_{2}$ molecule by the fixed nuclei R-matrix method as in our previous work on electron $\mathrm{O}_{2}$ scatterings [22, 23]. Although theoretical treatment is similar to the previous work of Gillan et al.[21], more target states and partial waves of a scattering electron are included in the present work. Main purpose of this work is comparison of ICSs as well as DCSs for the 8 lowest excited states with the experimental results of Cartwright et al. [11], Brunger and Teubner[12], Campbell et al.[13], Khakoo et al. [2] and Johnson et al.[3]. This is because previous theoretical works have covered only a part of these 8 excitations.

In this paper, details of the calculation are presented in section 2, and we discuss the results in section 3 comparing our ICSs and DCSs with the previous theoretical and available experimental data. Then summary is given in section 4 . 


\section{THEORETICAL METHODS}

The R-matrix method itself has been described extensively in the literature [24, 25, 26] as well as in our previous paper[22]. Thus we do not repeat general explanation of the method here. We used a modified version of the polyatomic programs in the UK molecular R-matrix codes [24]. These programs utilize the gaussian type orbitals (GTO) to represent target electronic states as well as a scattering electron. Although most of the previous R-matrix works in electron $\mathrm{N}_{2}$ collisions have employed Slater type orbitals (STO), we select GTO mainly because of simplicity of the input and availability of basis functions. In the R-matrix calculations, we have included 13 target states; $X^{1} \Sigma_{g}^{+}, A^{3} \Sigma_{u}^{+}, B^{3} \Pi_{g}, W^{3} \Delta_{u}, B^{\prime 3} \Sigma_{u}^{-}, a^{\prime 1} \Sigma_{u}^{-}, a^{1} \Pi_{g}, w^{1} \Delta_{u}, C^{3} \Pi_{u}, E^{3} \Sigma_{g}^{+}, a^{\prime \prime 1} \Sigma_{g}^{+}, c^{1} \Pi_{u}$ and $c^{\prime 1} \Sigma_{u}^{+}$. The potential energy curves of these target electronic states are shown in figure 1 for reference. These target states were represented by valence configuration interaction wave functions constructed by state averaged complete active space SCF (SA-CASSCF) orbitals. Note that some target states, $E^{3} \Sigma_{g}^{+}, a^{\prime \prime} \Sigma_{g}^{+}$and $c^{\prime 1} \Sigma_{u}^{+}$, are Rydberg states and cannot be described adequately in the present valence active space. Inclusion of these states are intended to improve quality of the R-matrix calculations by adding more target states in the model, as in our previous works [22, 23] as well as other R-matrix works [27, 28]. Test calculation was performed with an extra $4 a_{g}$ orbital in the target orbital set. However, the target excitation energies as well as the excitation cross sections did not change much compared to the results with valence orbital set described above. Also, removal of $3 b_{1 u}$ orbital from target active space did not affect the result much in our calculation. In this study, the SA-CASSCF orbitals were obtained by calculations with MOLPRO suites of programs [29]. The target orbitals were constructed from the [5s $3 \mathrm{p} 1 \mathrm{~d}]$ level of basis set taken from Sarpal et al. [30]. In our fixed-bond R-matrix calculations, the target states were evaluated at the equilibrium bond length $R=2.068 \mathrm{a}_{0}$ of the $\mathrm{N}_{2} X^{1} \Sigma_{g}^{+}$ground electronic state. Although we also performed calculations with $R=2.100 \mathrm{a}_{0}$ as in the previous R-matrix calculation of Gillan et al.[21], the cross sections with $R=2.068 \mathrm{a}_{0}$ and $R=2.100 \mathrm{a}_{0}$ are almost the same. Thus, we will only show the results with the equilibrium bond length of $\mathrm{N}_{2}$ in the next section. The radius of the R-matrix sphere was chosen to be $10 \mathrm{a}_{0}$ in our calculations. In order to represent the scattering electron, we included diffuse gaussian functions up to $l=5$, with 9 functions for $l=0,7$ functions for $l=1-3$ and 6 functions for $l=4$ and 5. Exponents of these diffuse gaussians were fitted using the GTOBAS program [31] in the UK R-matrix codes. In addition to these continuum orbitals, we included 8 extra virtual orbitals, one for each symmetry. 
We constructed the 15-electron configurations from the orbitals listed in table 【. The CI target wave functions are composed of the valence orbitals in table $\prod$ with the $1 a_{g}$ and $1 b_{1 u}$ orbitals kept doubly occupied. We have included 3 types of configurations in the calculation. The first type of configurations has the form,

$$
1 a_{g}^{2} 1 b_{1 u}^{2}\left\{2 a_{g} 3 a_{g} 1 b_{2 u} 1 b_{3 u} 2 b_{1 u} 3 b_{1 u} 1 b_{3 g} 1 b_{2 g}\right\}^{10}\left({ }^{1} A_{g}\right)\left\{5 a_{g} \ldots 39 a_{g}\right\}^{1}\left({ }^{2} A_{g}\right),
$$

here we assume that the total symmetry of this 15 electrons system is ${ }^{2} A_{g}$. The first 4 electrons are always kept in the $1 a_{g}$ and $1 b_{1 u}$ orbitals, then the next 10 electrons are distributed over the valence orbitals with restriction of target state symmetry, ${ }^{1} A_{g}$ symmetry of the $\mathrm{N}_{2}$ ground state in this case. The last electron, the scattering electron, occupies one of the diffuse orbitals, of $a_{g}$ symmetry in this example. To complete the wave function with the total symmetry ${ }^{2} A_{g}$, we also have to include configurations with the other target states combined with diffuse orbitals having appropriate symmetry in the same way as in the example. The second type of configurations has the form,

$$
1 a_{g}^{2} 1 b_{1 u}^{2}\left\{2 a_{g} 3 a_{g} 1 b_{2 u} 1 b_{3 u} 2 b_{1 u} 3 b_{1 u} 1 b_{3 g} 1 b_{2 g}\right\}^{10}\left({ }^{1} A_{g}\right)\left\{4 a_{g}\right\}^{1}\left({ }^{2} A_{g}\right),
$$

where the scattering electron occupies a bound $4 a_{g}$ extra virtual orbital, instead of the diffuse continuum orbitals in the expression (1). As in table I we included one extra virtual orbital for each symmetry. The third type of configurations has the form,

$$
1 a_{g}^{2} 1 b_{1 u}^{2}\left\{2 a_{g} 3 a_{g} 1 b_{2 u} 1 b_{3 u} 2 b_{1 u} 3 b_{1 u} 1 b_{3 g} 1 b_{2 g}\right\}^{11}\left({ }^{2} A_{g}\right) .
$$

In this case, the last 11 electrons including the scattering electron are distributed over the valence orbitals with the restriction of ${ }^{2} A_{g}$ symmetry. Note that the third type of configurations are crucial in description of $\mathrm{N}_{2}^{-}$resonance states, which often have dominant contributions to the excitation cross sections. In this way, the number of configurations generated for a specific total symmetry is typically about 60000 , though the final dimension of the inner region Hamiltonian is reduced to be about 600 by using CI target contraction and prototype CI expansion method [32].

The R-matrix calculations were performed for all 8 irreducible representations of the $\mathrm{D}_{2 h}$ symmetry, $A_{g}, B_{2 u}, B_{3 u}, B_{1 g}, B_{1 u}, B_{3 g}, B_{2 g}$ and $A_{u}$, in doublet spin multiplicity of the electron plus target system. DCSs were evaluated in the same way as in our previous paper[23]. 


\section{RESULTS AND DISCUSSION}

\section{A. Excitation energies}

Figure 1 shows the potential energy curves of all $\mathrm{N}_{2}$ target states included in the present Rmatrix model. These curves were obtained by the same SA-CASSCF method employed in our R-matrix calculation. Table $\amalg$ compares the excitation energies of the $\mathrm{N}_{2}$ target states from the present calculation with the previous R-matrix results of Gillan et al.[21], multi-reference coupled cluster results of Ben-Shlomo and Kaldor [33] as well as experimental values. Since these energies are evaluated at different inter-nuclear distance, $2.068 a_{0}$ in our case, $2.100 a_{0}$ in Gillan et al.[21] and $2.074 a_{0}$ in Ben-Shlomo and Kaldor [33], precise comparison is not so meaningful. However, deviations of excitation energies from the experimental values are less than $0.8 \mathrm{eV}$ in our calculation, which is good considering the level of calculation. In terms of excitation energies, our calculation and the previous R-matrix calculation of Gillan et al.[21] have similar quality.

In addition to this good agreement of target energies with experimental results, $\mathrm{N}_{2}^{+}$energies are also well described in our SA-CASSCF calculation. In our calculation, $\mathrm{N}_{2}^{+} X^{2} \Sigma_{g}^{+}$and $A^{2} \Pi_{u}$ states are located at 15.63 and $17.21 \mathrm{eV}$ above $\mathrm{N}_{2} X^{1} \Sigma_{g}^{+}$state, respectively. Compared to the experimental values of 15.61 and $17.08 \mathrm{eV}$, our SA-CASSCF calculation gives good results. Note that the energy ordering of $\mathrm{N}_{2}^{+} X^{2} \Sigma_{g}^{+}$and $A^{2} \Pi_{u}$ states are not well described in the Hartree Fock level calculation, see Ermler and McLean [34] for example.

\section{B. Integral cross sections}

Figure 2 shows integral cross sections for electron impact excitation from the $\mathrm{N}_{2} X^{1} \Sigma_{g}^{+}$state to the $A^{3} \Sigma_{u}^{+}, B^{3} \Pi_{g}, W^{3} \Delta_{u}$ and $B^{\prime 3} \Sigma_{u}^{-}$states. In this figure, present results are compared with the previous R-matrix calculations of Gillan et al.[21], recent calculations of da Costa and Lima [35], experimental results of Cartwright et al. [11], Campbell et al.[13] and recent measurements of Johnson et al.[3]. Renormalized values of Cartwright et al. [11] are used as recommended by Trajmar et al. [36]. Figure 3 compares the present excitation cross sections of the $a^{\prime 1} \Sigma_{u}^{-}, a^{1} \Pi_{g}$, $w^{1} \Delta_{u}$ and $C^{3} \Pi_{u}$ states with the previous experimental results of Cartwright et al. [11], Campbell et al.[13] and Johnson et al.[3]. For the $a^{1} \Pi_{g}$ state cross sections, the recent calculations of da Costa and Lima [35], other experimental values of Ajello and Shemansky [5], Zetner and Trajmar [10] and Mason and Newell [6] are included. For the $C^{3} \Pi_{u}$ state cross sections, the experimental 
results of Zubek [8], Zubek and King [9] and Poparic et al. [7] are included.

Our excitation cross sections for the $A^{3} \Sigma_{u}^{+}$state have a resonance feature at approximately $12 \mathrm{eV}$ as in the previous R-matrix results of Gillan et al.[21]. The $\mathrm{N}_{2}^{-}{ }^{2} \Pi_{u}$ resonance state is responsible for this peak structure. The main configuration of this resonance state is $1 \pi_{u}^{3} 1 \pi_{g}^{2}$. Other than the ${ }^{2} \Pi_{u}$ symmetry partial cross sections, The ${ }^{2} \Pi_{g}$ symmetry contributes to the ICSs as a smooth background component (not shown in the figure). Compared to the previous Rmatrix cross sections, the peak at $12 \mathrm{eV}$ is more pronounced in our case. Our results are slightly larger than theirs at 12-17.5 eV. Compared to the recent experimental results of Johnson et al.[3], our cross sections are about $50 \%$ larger at $12.5-20 \mathrm{eV}$, though $50 \%$ smaller at $10 \mathrm{eV}$. Also our calculation overestimates the results of Campbell et al.[13], however, the results of Cartwright et al. [11] agree well with our results except at $12.5 \mathrm{eV}$. The position of the resonance peak depends rather strongly on the inter-nuclear distance of $\mathrm{N}_{2}$ molecule, which is $12.2 \mathrm{eV}$ for $2.068 a_{0}$ and $11.75 \mathrm{eV}$ for $2.100 a_{0}$ in our calculations. Thus, inclusion of vibrational motion may be necessary to resolve this discrepancy of the resonance peak.

Our excitation cross sections for the $B^{3} \Pi_{g}$ state have a small bump at $12.8 \mathrm{eV}$, which is not evident in the previous R-matrix cross sections. The origin of this bump is the $\mathrm{N}_{2}^{-} 1^{2} \Delta_{g}$ state, with main configuration of $3 \sigma_{g}^{1} 1 \pi_{g}^{2}$. Other than this bump, the ICSs are mostly composed of the ${ }^{2} \Pi_{g}$ symmetry contribution and have a shape similar to the previous R-matrix results. The magnitude of our ICSs is about 50\% larger than the previous results of Gillan et al.[21]. Recently, da Costa and Lima [35] calculated ICSs for the $B^{3} \Pi_{g}$ state using the Schwinger multichannel method with the minimal orbital basis for the single configuration interactions (MOB-SCI) approach. There cross sections are much larger than our results above $12 \mathrm{eV}$. Also, there is a prominent peak around 10 $\mathrm{eV}$ in their ICSs, which does not exist in the R-matrix calculations. Compared to the experimental ICSs, our results agree well with the cross sections of Cartwright et al. [11], especially above 15 eV. However, the results of Campbell et al.[13] are much larger than ours. Recent measurements of Johnson et al.[3] agree better with the previous R-matrix calculation of Gillan et al.[21].

For the excitation cross sections for the $W^{3} \Delta_{u}$ state, our results have a shape and magnitude similar to the previous R-matrix results. Most of our ICSs are composed of the ${ }^{2} \Pi_{g}$ symmetry partial cross sections. Agreement with the experimental cross sections of Johnson et al.[3] is good in this case. The cross sections of Campbell et al.[13] agree well with our results at 15 and 17.5 $\mathrm{eV}$, but their value is about half as much as our result at $20 \mathrm{eV}$. The results of Cartwright et al. [11] are about two times larger than our cross sections. 
Our excitation cross sections for the $B^{\prime 3} \Sigma_{u}^{-}$state are about half of the previous R-matrix cross sections of Gillan et al.[21]. Apart from this difference in magnitude, the shape of the cross sections is similar. Dominant component in these ICSs is the ${ }^{2} \Pi_{g}$ symmetry partial cross sections, although the ${ }^{2} \Pi_{u}$ symmetry also has certain contribution around 18-20 eV. Among 3 different experimental measurements, our results agree well with the results of Johnson et al.[3]. The experimental cross sections of other two groups are much larger than our results at 15 and $17.5 \mathrm{eV}$, and have a different energy dependence compared to the present calculation.

The situation of the excitation cross sections for the $a^{\prime 1} \Sigma_{u}^{-}$state is similar to the case of the $B^{\prime 3} \Sigma_{u}^{-}$state. The ${ }^{2} \Pi_{g}$ and ${ }^{2} \Pi_{u}$ symmetry partial cross sections contribute almost equally to the ICSs. Our cross sections roughly agree with the results of Johnson et al.[3], while the cross sections of Cartwright et al. [11] and Campbell et al.[13] at $15 \mathrm{eV}$ are much larger than our result. The results of Cartwright et al. [11] and Campbell et al.[13] decrease as impact energy increases from 15 to $20 \mathrm{eV}$, however, our cross sections increase mildly in this energy region.

In case of excitation to the $a^{1} \Pi_{g}$ state, several other experimental results are available in addition to the measurements of Cartwright et al. [11], Campbell et al.[13], and Johnson et al.[3]. The cross section profiles of Johnson et al.[3], Ajello and Shemansky [5], Cartwright et al. [11] and Mason and Newell [6] are similar to our ICSs. However, the magnitude of our cross sections is lower than the experimental values in most case except the cross sections of Johnson et al.[3]. At 15, 17.5 and $20 \mathrm{eV}$, agreement of our results with the cross sections of Johnson et al.[3] is very good, although our cross section at $12.5 \mathrm{eV}$ is twice as large as their value. Note that there is no dominant symmetry contribution to the calculated ICSs. All partial cross sections contribute rather equally to the ICSs. Recent ICSs of da Costa and Lima [35] by the Schwinger multichannel method are also shown in the panel (b) of the figure 3. Their result has a sharp peak at $12 \mathrm{eV}$ as in their calculation for the $B^{3} \Pi_{g}$ state excitation. This difference between our and their results may come from different number of target states considered in the scattering calculation. Only the $X^{1} \Sigma_{g}^{1}$, $a^{1} \Pi_{g}$ and $B^{3} \Pi_{g}$ states were included in the calculations of da Costa and Lima. The other part of the cross section profile is similar to the shape of our cross sections, although the magnitude of their cross sections are about twice as large as our results at $15-20 \mathrm{eV}$.

Our excitation cross section for the $w^{1} \Delta_{u}$ state gradually increases as a function of energy from the threshold to the broad peak around $17.5 \mathrm{eV}$, then decreases toward $20 \mathrm{eV}$. In this case, agreement with the results of Johnson et al.[3] is not so good compared to the excitations of the $a^{1} \Pi_{g}$ and $a^{\prime 1} \Sigma_{u}^{-}$states. Our cross sections are about $50 \%$ larger than their values at 17.5 and $20 \mathrm{eV}$. 
At $15 \mathrm{eV}$, our results agree well with the cross section of Johnson et al. [3], however, they are about $50 \%$ lower than the results of Cartwright et al. [11] and Campbell et al.[13]. In the calculated ICSs, the ${ }^{2} \Pi_{u}$ symmetry partial cross section is a major component, with a minor contribution from the ${ }^{2} \Pi_{g}$ symmetry.

The calculated excitation cross sections for the $C^{3} \Pi_{u}$ state has a peak similar to the experimental results of Zubek [8] and Poparic et al.[7]. Although the shape of the cross sections is similar, position of the cross section peak is different from experimental results. In our case, it is located at about $17 \mathrm{eV}$, whereas corresponding peaks are located at $14 \mathrm{eV}$ in the experimental cross sections. The height of the peak in our ICSs is lower than the experimental values of Zubek [8] and Poparic et al.[7]. It is unclear whether there is a cross section peak in the experimental cross sections of Cartwright et al. [11], Campbell et al.[13] and Johnson et al.[3]. At least, it appears that they do not have a peak around $17 \mathrm{eV}$. The origin of this discrepancy in the cross section peak is uncertain, but may be related to the employment of the fixed-nuclei approximation or insufficiency of higher excited target states in the R-matrix model. The calculated ICSs are composed of the ${ }^{2} \Sigma_{u}^{+}$and ${ }^{2} \Sigma_{u}^{-}$symmetry partial cross sections near the peak structure at $17 \mathrm{eV}$. The contribution of the ${ }^{2} \Sigma_{u}^{+}$ symmetry is about $50 \%$ larger than the ${ }^{2} \Sigma_{u}^{-}$component. Other than these two symmetries, the ${ }^{2} \Pi_{g}$ symmetry partial cross section contributes to the ICSs as a smooth background component.

\section{Differential cross sections}

Figure 4 shows calculated DCSs for excitation of the $A^{3} \Sigma_{u}^{+}$state with the experimental results of Khakoo et al. [2], Brunger and Teubner [12], Cartwright et al. [11], Zetner and Trajmar [10], LeClair and Trajmar [37] and the previous R-matrix DCSs of Gillan et al.[21]. Our DCSs at 12.5, 15 and $17.5 \mathrm{eV}$ have similar shape in common. They are enhanced in backward direction and have a small dimple at 120 degrees with a bump at 75 degrees. At $17.5 \mathrm{eV}$, our cross sections are located between the experimental values of Khakoo et al.[2] and Cartwright et al. [11]. The profile of the experimental DCSs are reproduced well in our calculation. At $15 \mathrm{eV}$, our results agree better with the results of Khakoo et al. [2] compared to the other experiments. In the DCSs of the previous R-matrix calculation of Gillan et al.[21], a bump is located at 40 degrees and a small dimple is located at 100 degrees, which agree better with the experimental results of Brunger and Teubner[12]. In our calculation, these dimple and bump are shifted toward backward direction by 20 degrees, and agreement with the results of Brunger and Teubner [12] is not so good. At 12.5 
$\mathrm{eV}$, our calculation overestimates the experimental results by a factor of two. As seen in panel (a) of Fig.2, this discrepancy is related to the existence of a resonance peak around $12.5 \mathrm{eV}$.

Figure 5 compares calculated excitation DCSs for the $B^{3} \Pi_{g}$ state with the experimental and recent theoretical results. Our DCSs at 12.5, 15 and $17.5 \mathrm{eV}$ have backward-enhanced feature with a broad peak at 130 degrees. At 15 and $17.5 \mathrm{eV}$, our DCSs agree well with the results of Khakoo et al. [2] at forward direction below 80 degrees. However, their DCSs are smaller than ours by a factor of two at 80-130 degrees. Agreement with the results of Cartwright et al. [11] at $15 \mathrm{eV}$ is good at 20-130 degrees, although their DCSs are twice as large as our DCSs at $17.5 \mathrm{eV}$ for low scattering angles. Because of a resonance-like feature at $12.5 \mathrm{eV}$ as seen in panel (b) of Fig.2, our results are larger than the experimental results at $12.5 \mathrm{eV}$. Recent Schwinger multi-channel results of da Costa and Lima [35] are much larger than our DCSs at 12.5 and $15 \mathrm{eV}$. The deviation is especially large at $12.5 \mathrm{eV}$, which is possibly related to the difference in the excitation energies of the target state.

Figure 6 shows the excitation DCSs for the $W^{3} \Delta_{u}$ state with the experimental cross sections. At 15 and $17.5 \mathrm{eV}$, our cross section gradually increases as a function of scattering angle, without noticeable bump or dip. At $12.5 \mathrm{eV}$, the shape of DCSs is nearly symmetric around 90 degrees. Agreement with the experimental DCSs of Khakoo et al. [2] is good, although their results at 15 and $17.5 \mathrm{eV}$ have more complex structure such as a small peak at 80 degrees. Our DCSs are generally smaller than the other experimental results of Brunger and Teubner[12], Cartwright et al. [11], Zetner and Trajmar [10].

Excitation cross sections for the $B^{\prime 3} \Sigma_{u}^{-}$state are shown in figure 7. Calculated DCSs decrease to be zero toward 0 and 180 degrees, because of a selection rule associated with $\Sigma^{+}-\Sigma^{-}$transition [38, 39]. Our DCSs have a broad single peak near 90 degrees at 12.5 and $15 \mathrm{eV}$, whereas there are two broad peaks at $17.5 \mathrm{eV}$. The position of the right peak at $17.5 \mathrm{eV}$ coincides with that of the experimental DCSs of Khakoo et al. [2] and Cartwright et al. [11], although the peak of Cartwright et al. [11] is much higher than ours. Our results agree well with the DCSs of Khakoo et al. [2] at 15 and $17.5 \mathrm{eV}$. However, their cross sections at $15 \mathrm{eV}$ have a small dip at 100 degrees and a small bump 60 degrees, which do not exist in our results. At $12.5 \mathrm{eV}$, our cross sections are slightly larger than the results of Khakoo et al.[2]. On the whole, agreement with the other experimental results of Brunger and Teubner [12] and Cartwright et al. [11] is not good.

Figure 8 shows the excitation DCSs for the $a^{\prime 1} \Sigma_{u}^{-}$state. Because of $\Sigma^{+}-\Sigma^{-}$selection rule, DCSs at 0 and 180 degrees become zero as in the case of the $B^{\prime 3} \Sigma_{u}^{-}$state DCSs. Calculated DCSs have a 
broad single peak near 60 degrees at 12.5 and $15 \mathrm{eV}$. At $17.5 \mathrm{eV}$, there are two broad peaks at 50 and 120 degrees. Although there is slight overestimation of DCSs near 50-60 degrees, our DCSs agree marginally with the results of Khakoo et al.[2]. Agreement with the other experimental results is not good except low scattering angles at $17.5 \mathrm{eV}$.

Figure 9 compares our excitation DCSs for the $a^{1} \Pi_{g}$ state with the experimental cross sections. Because of large variation of the DCSs, the cross sections are shown in logarithmic scale. Calculated DCSs are strongly forward-enhanced, which is consistent with all experimental results shown in the figure. Our DCSs at $12.5 \mathrm{eV}$ have a small dip around 100 degrees, which moves forward to 85 degrees at $15 \mathrm{eV}$ and 75 degrees at $17.5 \mathrm{eV}$. This behavior roughly agrees with the results of Cartwright et al.[11] and Khakoo et al.[2]. At $15 \mathrm{eV}$, our DCSs agree better with the results of Khakoo et al.[2] than the other experimental DCSs. At $17.5 \mathrm{eV}$, the results of Cartwright et al.[11] are closer to our DCSs at scattering angles above 40 degrees. Below 40 degrees, our calculation significantly underestimates the experimental DCSs. Our results at $12.5 \mathrm{eV}$ are located between the DCSs of Cartwright et al. [11] and Khakoo et al.[2], however the shape of the DCSs is similar to their results. The shapes of DCSs calculated by da Costa and Lima [35] are similar to our results. However, their cross sections are larger than our results at low-scattering angles below 80 degrees, where their results agree better with the experimental DCSs of Brunger and Teubner [12] and Zetner and Trajmar [10].

Figure 10 shows calculated excitation DCSs for the $w^{1} \Delta_{u}$ state with the experimental cross sections. Our DCSs are enhanced in forward direction as in the case of the $a^{1} \Pi_{g}$ state. However, magnitude of the enhancement is much smaller than that of the $a^{1} \Pi_{g}$ state. Agreement with the DCSs of Cartwright et al. [11] is good at $17.5 \mathrm{eV}$ except low scattering angles below 20 degrees. At 12.5 and $15 \mathrm{eV}$, their results are much larger than our DCSs. At $15 \mathrm{eV}$, our DCSs agree marginally with the results of Khakoo et al. [2], although details of the DCS profile are different. Their results are smaller than ours at 17.5 and $12.5 \mathrm{eV}$. Discrepancy is especially large for forward scattering at $12.5 \mathrm{eV}$.

Figure 11 shows excitation DCSs for the $C^{3} \Pi_{u}$ state with the experimental cross sections of Khakoo et al.[2], Brunger and Teubner[12], Zubek and King [9] and Cartwright et al. [11]. Calculated DCS profiles are almost flat at 12.5 and $15 \mathrm{eV}$, whereas they are enhanced in backward direction at $17.5 \mathrm{eV}$. Below 90 degrees, slope of the calculated DCSs at $17.5 \mathrm{eV}$ is similar to the results of Khakoo et al.[2], Zubek and King [9] and Cartwright et al. [11], though our results are about 50\% larger than their DCSs. In general, our results do not agree well with the experimental 
DCSs. Although the ICS of Khakoo et al. [2] at $15 \mathrm{eV}$ agrees well our result as shown in panel (d) of Fig, 3, angular dependence of the cross sections appears to be different.

\section{Discussion}

The excitation ICSs of the $B^{3} \Pi_{g}$ state, shown in panel (b) of figure 2, have a small bump around $13 \mathrm{eV}$. However, there is no such structure in the previous R-matrix ICSs of Gillan et al. [21]. The origin of this bump in our calculation is the $\mathrm{N}_{2}^{-} 1^{2} \Delta_{g}$ state, with main configuration of $3 \sigma_{g}^{1} 1 \pi_{g}^{2}$. The existence of the $\mathrm{N}_{2}^{-} 1^{2} \Delta_{g}$ state can also be verified by usual CASSCF calculation on $\mathrm{N}_{2}^{-}$with valence active space ignoring continuum orbitals. In molpro calculation, the energy of the $1^{2} \Delta_{g}$ state is $15.7 \mathrm{eV}$. Since diffuse continuum orbitals are added in the R-matrix calculation, the energy of the state is stabilized to be $12.8 \mathrm{eV}$ in the present scattering calculation. In the same way, the $\mathrm{N}_{2}^{-}{ }^{2} \Pi_{u}\left(1 \pi_{u}^{3} 1 \pi_{g}^{2}\right)$ resonance peak in the $A^{3} \Sigma_{u}^{+}$excitation ICSs can be verified by usual CASSCF calculation. In molpro calculation, it is located at $14.7 \mathrm{eV}$, whereas the position of the resonance is stabilized to be $12.2 \mathrm{eV}$ in our R-matrix scattering calculation. It is unclear why the bump in the ICSs of the $B^{3} \Pi_{g}$ state is not evident in the previous R-matrix cross sections of Gillan et al.[21]. Some details of the R-matrix calculations are different in their calculation and ours, e.g., they used hybrid orbitals with Slater type functions, whereas we employed SA-CASSCF orbitals with Gaussian type functions. These difference may contribute to the difference in magnitude of the ${ }^{2} \Delta_{g}$ partial cross section.

In this study, we employed the fixed-nuclei (FN) approximation. As we can see in figure 1 , equilibrium bond lengths of the excited $\mathrm{N}_{2}$ states are longer than that of the ground state. Thus, in principle, it would be desirable to include the effect of nuclear motion in the R-matrix calculation. Use of the FN approximation may be responsible for several discrepancies between our calculation and experiments, including bumps in the ICSs of the $A^{3} \Sigma_{u}^{+}$and $B^{3} \Pi_{g}$ states, the position of the peak in the ICSs of the $C^{3} \Pi_{u}$ state. Although the calculated DCSs agree very well with experimental results in general, our DCSs of the $A^{3} \Sigma_{u}^{+}, B^{3} \Pi_{g}, w^{1} \Delta_{u}$ and $C^{3} \Pi_{u}$ states at $12.5 \mathrm{eV}$ are $2-4$ times larger than experimental results. These deviations in the near-threshold DCSs can also be related to the FN approximation. In spite of these discrepancies, good agreements are observed between our calculation and experiments in most ICS and DCS cases as we can see in the figures. Agreements with the recent experimental results of Khakoo et al.[2] and Johnson et al. [3] are especially impressive. It is possible to include nuclear motion in the R-matrix formalism through vibrational 
averaging of T-matrix elements or the non-adiabatic R-matrix method, though application of these methods will be a difficult task in the presence of many target electronic states. In the future, we plan to perform the R-matrix calculation with these methods including nuclear motion. 


\section{SUMMARY}

We have investigated electron impact excitations of $\mathrm{N}_{2}$ molecule using the fixed-bond R-matrix method which includes 13 target electronic states, $X^{1} \Sigma_{g}^{+}, A^{3} \Sigma_{u}^{+}, B^{3} \Pi_{g}, W^{3} \Delta_{u}, B^{\prime 3} \Sigma_{u}^{-}, a^{\prime 1} \Sigma_{u}^{-}, a^{1} \Pi_{g}$, $w^{1} \Delta_{u}, C^{3} \Pi_{u}, E^{3} \Sigma_{g}^{+}, a^{\prime \prime 1} \Sigma_{g}^{+}, c^{1} \Pi_{u}$ and $c^{\prime 1} \Sigma_{u}^{+}$. These target states are described by CI wave functions in the valence CAS space, using SA-CASSCF orbitals. Gaussian type orbitals were used in this work, in contrast to the STOs in the previous R-matrix works. We have obtained integral cross sections as well as differential cross sections of excitations to the $A^{3} \Sigma_{u}^{+}, B^{3} \Pi_{g}, W^{3} \Delta_{u}, B^{\prime} \Sigma_{u}^{-}, a^{\prime 1} \Sigma_{u}^{-}$, $a^{1} \Pi_{g}, w^{1} \Delta_{u}$ and $C^{3} \Pi_{u}$ states, which have been studied a lot experimentally but not enough theoretically before. In general, good agreements are observed both in the integrated and differential cross sections, which is encouraging for further theoretical and experimental studies in this field. However, some discrepancies are seen in the integrated cross sections of the $A^{3} \Sigma_{u}^{+}$and $C^{3} \Pi_{u}$ states, especially around a peak structure. Also, our DCSs do not agree well with the experimental results at low impact energy of $12.5 \mathrm{eV}$, compared to the higher energies of 15 and $17.5 \mathrm{eV}$. These discrepancies may be related to the fixed-nuclei approximation or insufficiency of higher excited target states in the R-matrix model.

\section{Acknowledgments}

The work of M.T. is supported by the Japan Society for the Promotion of Science Postdoctoral Fellowships for Research Abroad. The present research is supported in part by the grant from the Air Force Office of Scientific Research: the Advanced High-Energy Closed-Cycle Chemical Lasers project (PI: Wayne C. Solomon, University of Illinois, F49620-02-1-0357). 
[1] R. R. Meier, Space Science Rev. 58, 1 (1991).

[2] M. A. Khakoo, P. V. Johnson, I. Ozkay, P. Yan, S. Trajmar, and I. Kanik, Phys. Rev. A 71, 062703 (2005).

[3] P. V. Johnson, C. P. Malone, I. Kanik, K. Tran, and M. A. Khakoo, Journal of Geophysical Research (Space Physics) 110, 11311 (2005).

[4] R. P. Wayne, Chemistry of Atmospheres (Oxford University Press, New York, 2000), 3rd ed.

[5] J. M. Ajello and D. E. Shemansky, J. Geophysical Research-space Phys. 90, 9845 (1985).

[6] N. J. Mason and W. R. Newell, J. Phys. B-atomic Mol. Opt. Phys. 20, 3913 (1987).

[7] G. Poparic, M. Vicic, and D. S. Belic, Chem. Phys. 240, 283 (1999).

[8] M. Zubek, J. Phys. B-atomic Mol. Opt. Phys. 27, 573 (1994).

[9] M. Zubek and G. C. King, J. Phys. B-atomic Mol. Opt. Phys. 27, 2613 (1994).

[10] P. W. Zetner and S. Trajmar, in Abstracts of the Fifteenth International Conference on the Physics of Electronic and Atomic Collisions, Brighton, edited by J. Geddes, H. B. Gilbody, A. E. Kingston, and C. J. Latimer (Queen's University, Belfast, 1987), tabulated data was taken from Brunger and Buckman [15].

[11] D. C. Cartwright, A. Chutjian, S. Trajmar, and W. Williams, Phys. Rev. A 16, 1013 (1977).

[12] M. J. Brunger and P. J. O. Teubner, Phys. Rev. A 41, 1413 (1990).

[13] L. Campbell, M. J. Brunger, A. M. Nolan, L. J. Kelly, A. B. Wedding, J. Harrison, P. J. O. Teubner, D. C. Cartwright, and B. McLaughlin, J. Phys. B-atomic Mol. Opt. Phys. 34, 1185 (2001).

[14] Y. Itikawa, J. Phys. Chem. Reference Data 35, 31 (2006).

[15] M. J. Brunger and S. J. Buckman, Phys. Rep. 357, 215 (2002).

[16] S. Chung and C. C. Lin, Phys. Rev. A 6, 988 (1972).

[17] T. K. Holley, S. Chung, C. C. Lin, and E. T. P. Lee, Phys. Rev. A 24, 2946 (1981).

[18] A. W. Fliflet, V. Mckoy, and T. N. Rescigno, J. Phys. B-atomic Mol. Opt. Phys. 12, 3281 (1979).

[19] L. Mu-Tao and V. Mckoy, Phys. Rev. A 28, 697 (1983).

[20] C. J. Gillan, C. J. Noble, and P. G. Burke, J. Phys. B-atomic Mol. Opt. Phys. 23, L407 (1990).

[21] C. J. Gillan, J. Tennyson, B. M. McLaughlin, and P. G. Burke, J. Phys. B-atomic Mol. Opt. Phys. 29, 1531 (1996).

[22] M. Tashiro, K. Morokuma, and J. Tennyson, Phys. Rev. A 73, 052707 (2006). 
[23] M. Tashiro, K. Morokuma, and J. Tennyson, Phys. Rev. A 74, 022706 (2006).

[24] L. A. Morgan, J. Tennyson, and C. J. Gillan, Comput. Phys. Commun. 114, 120 (1998).

[25] P. G. Burke and J. Tennyson, Mol. Phys. 103, 2537 (2005).

[26] J. D. Gorfinkiel, A. Faure, S. Taioli, C. Piccarreta, G. Halmova, and J. Tennyson, Eur. Phys. J. D 35, 231 (2005).

[27] C. J. Noble and P. G. Burke, Phys. Rev. Lett. 68, 2011 (1992).

[28] K. Higgins, C. J. Noble, and P. G. Burke, J. Phys. B 27, 3203 (1994).

[29] H.-J. Werner, P. J. Knowles, R. Lindh, M. Schütz, et al., Molpro version 2002.6, a package of ab initio programs.

[30] B. K. Sarpal, K. Pfingst, B. M. Nestmann, and S. D. Peyerimhoff, J. Phys. B 29, 857 (1996).

[31] A. Faure, J. D. Gorfinkiel, L. A. Morgan, and J. Tennyson, Comput. Phys. Commun. 144, 224 (2002).

[32] J. Tennyson, J. Phys. B 29, 1817 (1995).

[33] S. B. Ben-Shlomo and U. Kaldor, J. Chem. Phys. 92, 3680 (1990).

[34] W. C. Ermler and A. D. McLean, J. Chem. Phys. 73, 2297 (1980).

[35] R. F. da Costa and M. A. P. Lima, International Journal of Quantum Chemistry 106, 2664 (2006).

[36] S. Trajmar, D. F. Register, and A. Chutjian, Phys. Reports-review Section Phys. Lett. 97, 221 (1983).

[37] L. R. LeClair and S. Trajmar, J. Phys. B-atomic Mol. Opt. Phys. 29, 5543 (1996).

[38] W. A. Goddard III, D. L. Huestis, D. C. Cartwright, and S. Trajmar, Chem. Phys. Lett. 11, 329 (1971).

[39] D. C. Cartwright, S. Trajmar, W. Williams, and D. L. Huestis, Phys. Rev. Lett. 27, 704 (1971). 


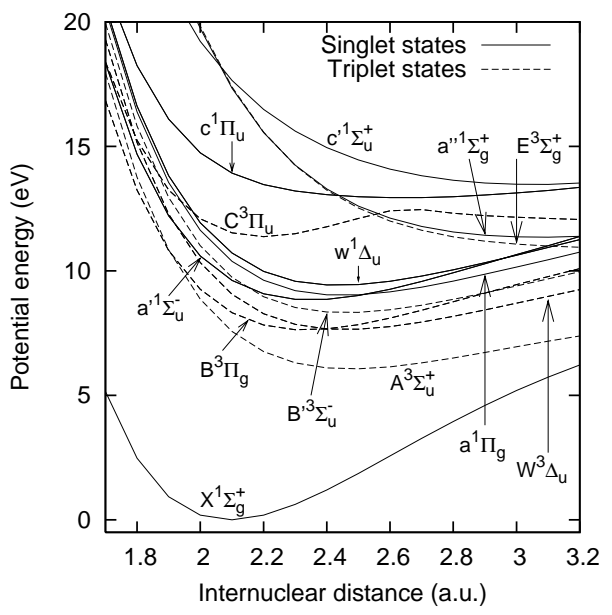

FIG. 1: Potential energy curves of the $\mathrm{N}_{2}$ electronic states. The equilibrium distance of the $X^{1} \Sigma_{g}^{+}$state, $R=$ $2.068 \mathrm{a}_{0}$ is used in our R-matrix calculations. 

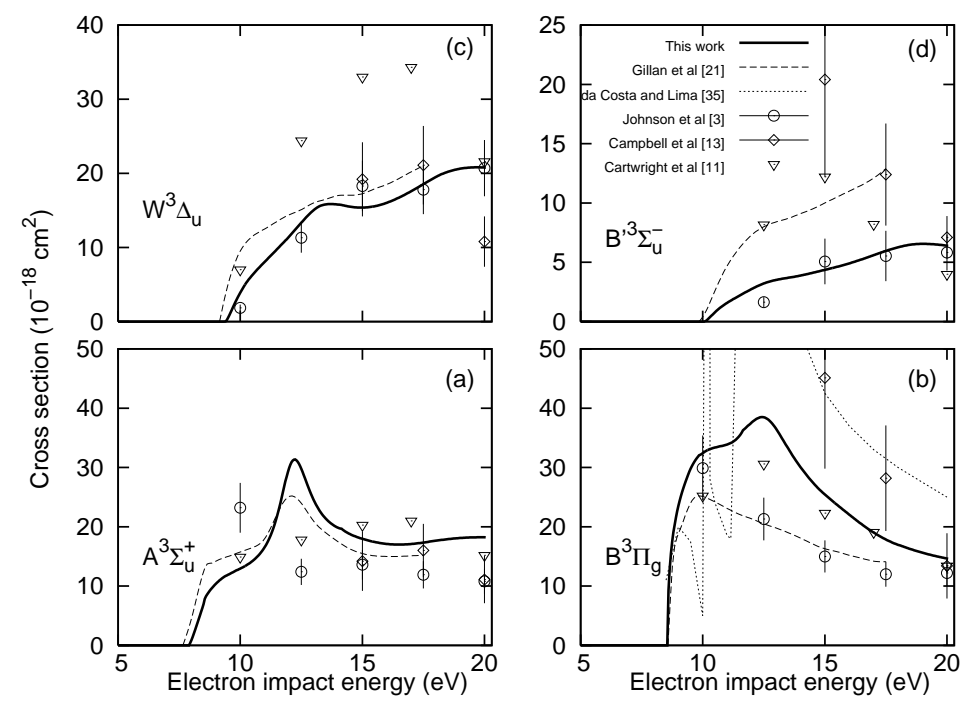

FIG. 2: Integral excitation cross sections of the $A^{3} \Sigma_{u}^{+}$(panel a), $B^{3} \Pi_{g}$ (panel b), $W^{3} \Delta_{u}$ (panel c) and $B^{\prime 3} \Sigma_{u}^{-}$ (panel d) states. Our results are shown in thick full lines. For comparison, we include the previous R-matrix results of Gillan et al. [21], Schwinger multichannel results of da Costa and Lima [35], the experimental cross sections of Cartwright et al. [11], Campbell et al.[13] and Johnson et al.[3].
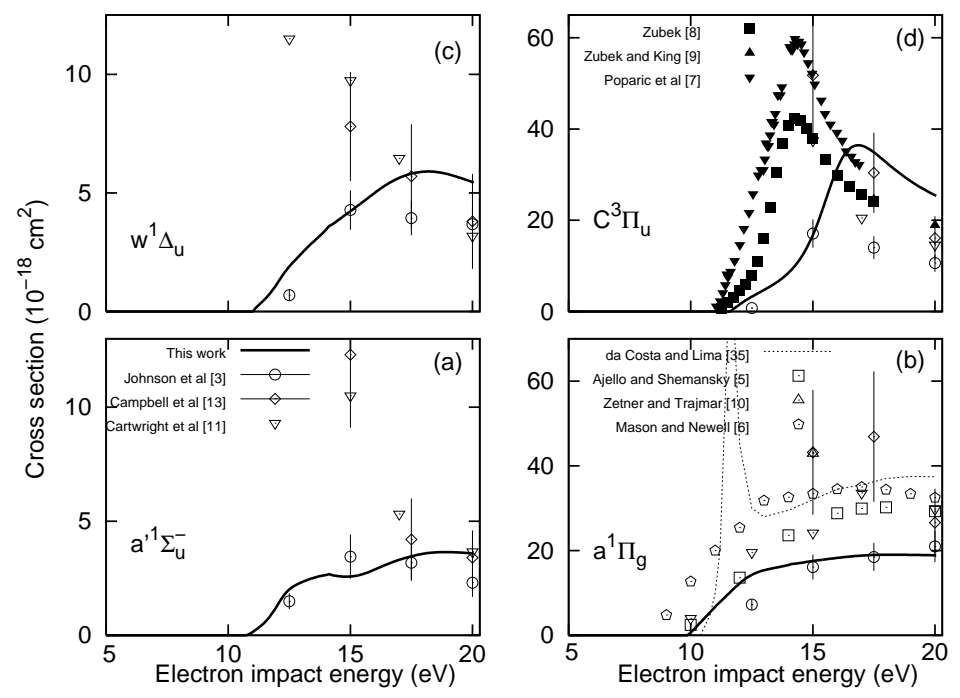

FIG. 3: Integral excitation cross sections of the $a^{\prime 1} \Sigma_{u}^{-}$(panel a), $a^{1} \Pi_{g}$ (panel b), $w^{1} \Delta_{u}$ (panel c) and $C^{3} \Pi_{u}$ (panel d) states. Our results are shown in thick full lines. In addition to the experimental ICSs in Fig,2, we include the results of Ajello and Shemansky [5], Zetner and Trajmar [10], Mason and Newell [6], Poparic et al. [7], Zubek [8] and Zubek and King [9]. 


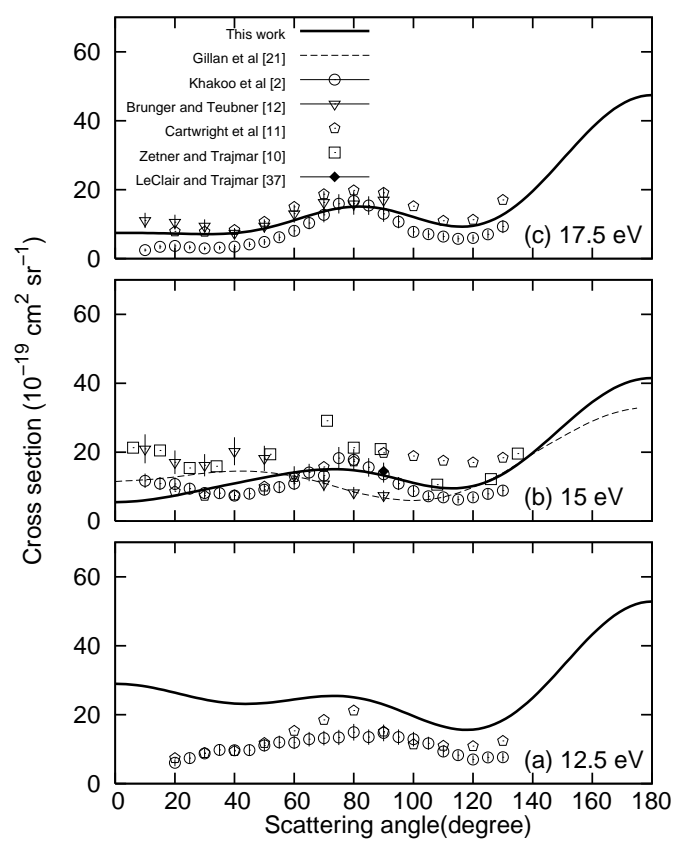

FIG. 4: Differential cross sections for electron impact excitation from the $\mathrm{N}_{2} X^{1} \Sigma_{g}^{+}$state to the $A^{3} \Sigma_{u}^{+}$state. Panel (a): electron impact energy of $12.5 \mathrm{eV}$, (b):15 eV and (c): $17.5 \mathrm{eV}$. Full line represents our result. For comparison, we include the previous theoretical cross sections of Gillan et al.[21], experimental results of Khakoo et al. [2], Brunger and Teubner [12], Cartwright et al. [11], Zetner and Trajmar [10] and LeClair and Trajmar [37]. 


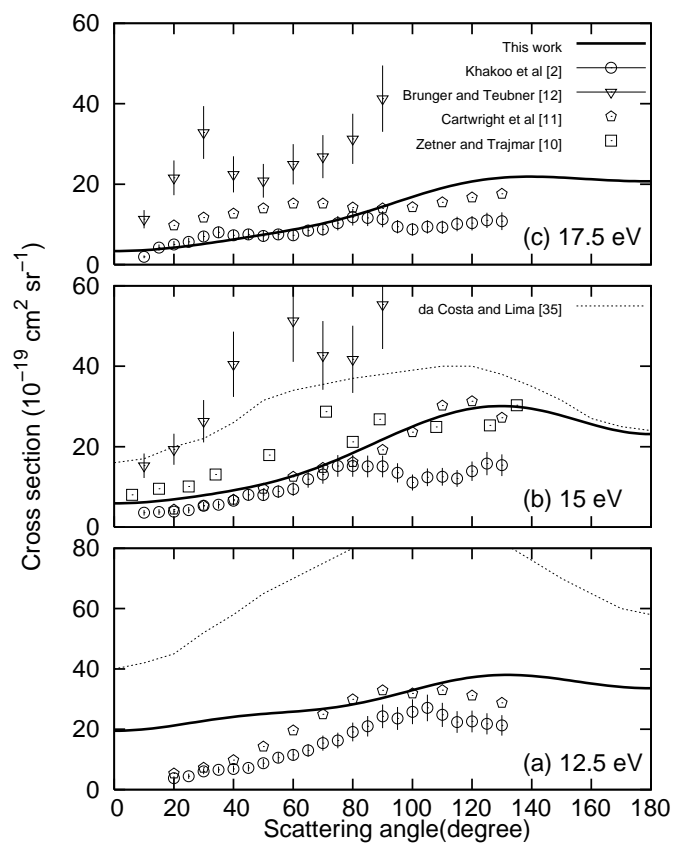

FIG. 5: Differential cross sections for electron impact excitation from the $\mathrm{N}_{2} X^{1} \Sigma_{g}^{+}$state to the $B^{3} \Pi_{g}$ state. Panel (a): electron impact energy of $12.5 \mathrm{eV}$, (b):15 eV and (c): $17.5 \mathrm{eV}$. The results of Schwinger multichannel calculation by da Costa and Lima [35] are also shown in the panels. Other details are the same as in Fig 4 


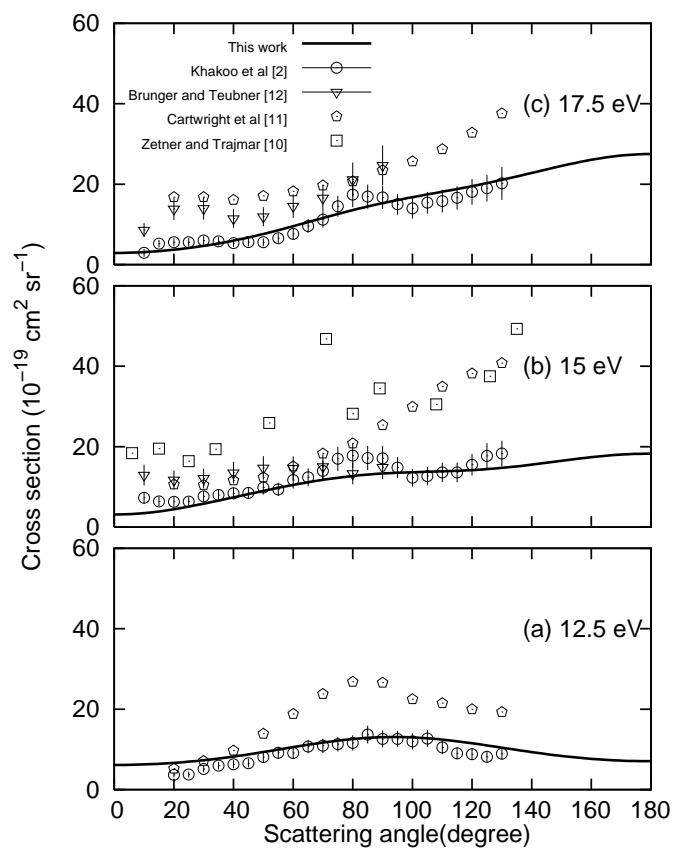

FIG. 6: Differential cross sections for electron impact excitation from the $\mathrm{N}_{2} X^{1} \Sigma_{g}^{+}$state to the $W^{3} \Delta_{u}$ state. Panel (a): electron impact energy of $12.5 \mathrm{eV}$, (b):15 eV and (c): $17.5 \mathrm{eV}$. Other details are the same as in Fig 4 


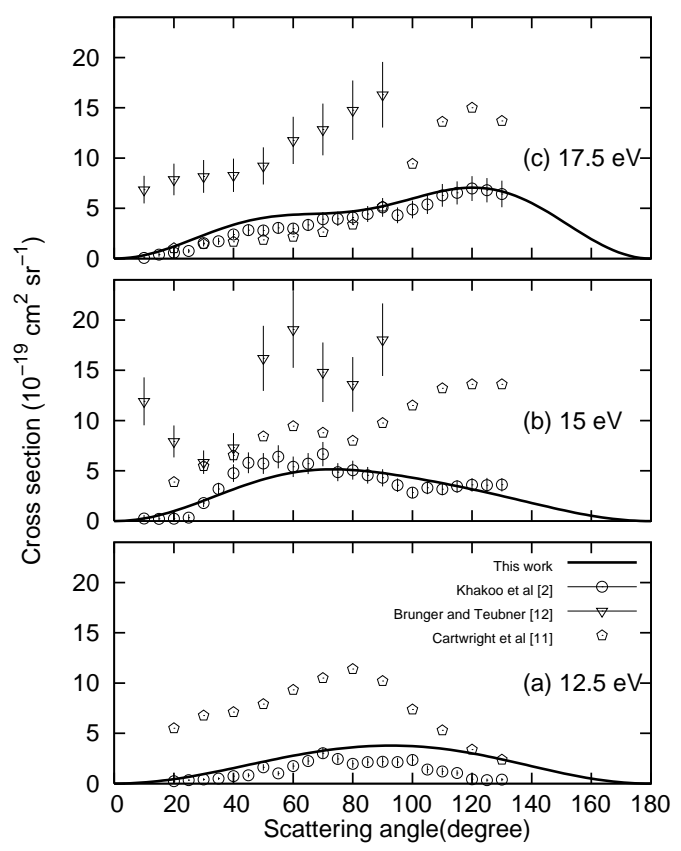

FIG. 7: Differential cross sections for electron impact excitation from the $\mathrm{N}_{2} X^{1} \Sigma_{g}^{+}$state to the $B^{\prime 3} \Sigma_{u}^{-}$state. Panel (a): electron impact energy of $12.5 \mathrm{eV},(\mathrm{b}): 15 \mathrm{eV}$ and (c): $17.5 \mathrm{eV}$. Other details are the same as in Fig 4 


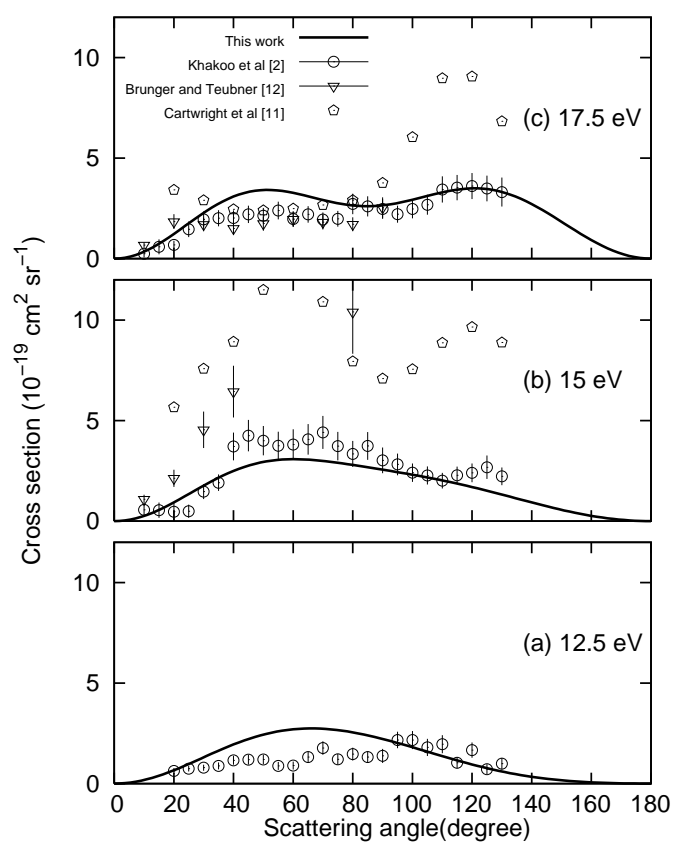

FIG. 8: Differential cross sections for electron impact excitation from the $\mathrm{N}_{2} X^{1} \Sigma_{g}^{+}$state to the $a^{\prime 1} \Sigma_{u}^{-}$state. Panel (a): electron impact energy of $12.5 \mathrm{eV},(\mathrm{b}): 15 \mathrm{eV}$ and (c): $17.5 \mathrm{eV}$. Other details are the same as in Fig 4 


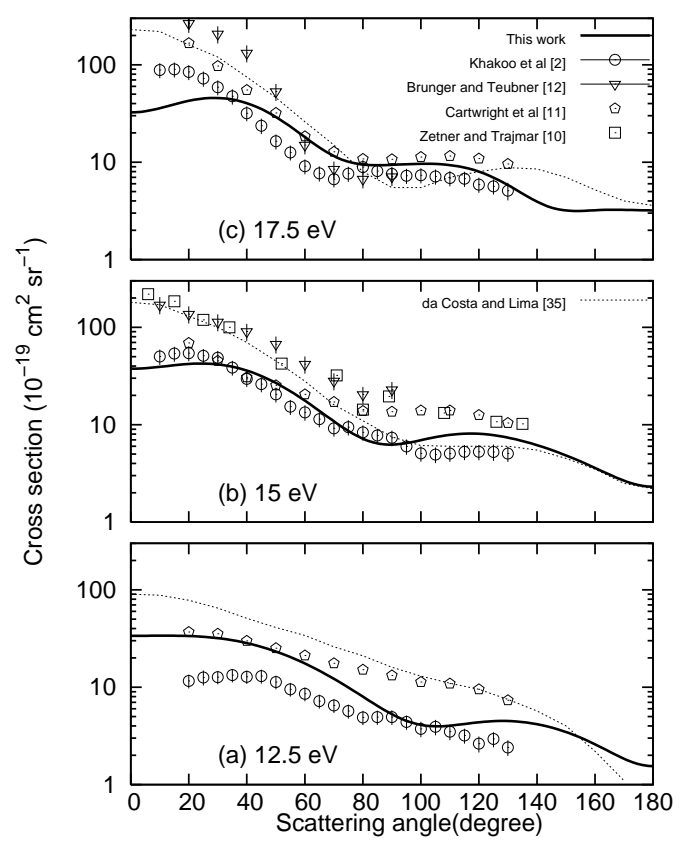

FIG. 9: Differential cross sections for electron impact excitation from the $\mathrm{N}_{2} X^{1} \Sigma_{g}^{+}$state to the $a^{1} \Pi_{g}$ state. Panel (a): electron impact energy of $12.5 \mathrm{eV},(\mathrm{b}): 15 \mathrm{eV}$ and (c): $17.5 \mathrm{eV}$. Note that the DCSs are shown in logarithmic scale. Other details are the same as in Fig, 4 


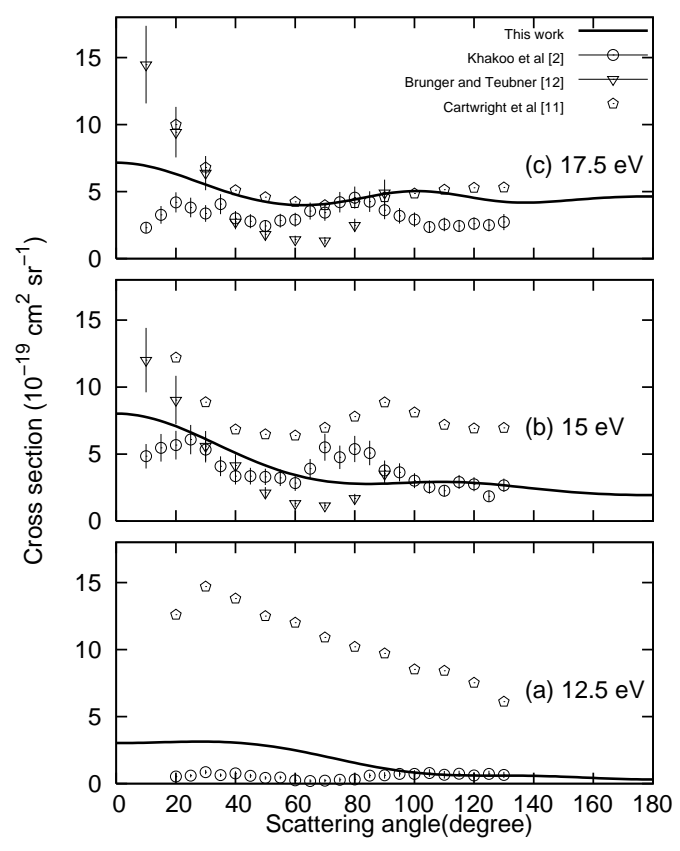

FIG. 10: Differential cross sections for electron impact excitation from the $\mathrm{N}_{2} X^{1} \Sigma_{g}^{+}$state to the $w^{1} \Delta_{u}$ state. Panel (a): electron impact energy of $12.5 \mathrm{eV},(\mathrm{b}): 15 \mathrm{eV}$ and (c): $17.5 \mathrm{eV}$. Other details are the same as in Fig 4 


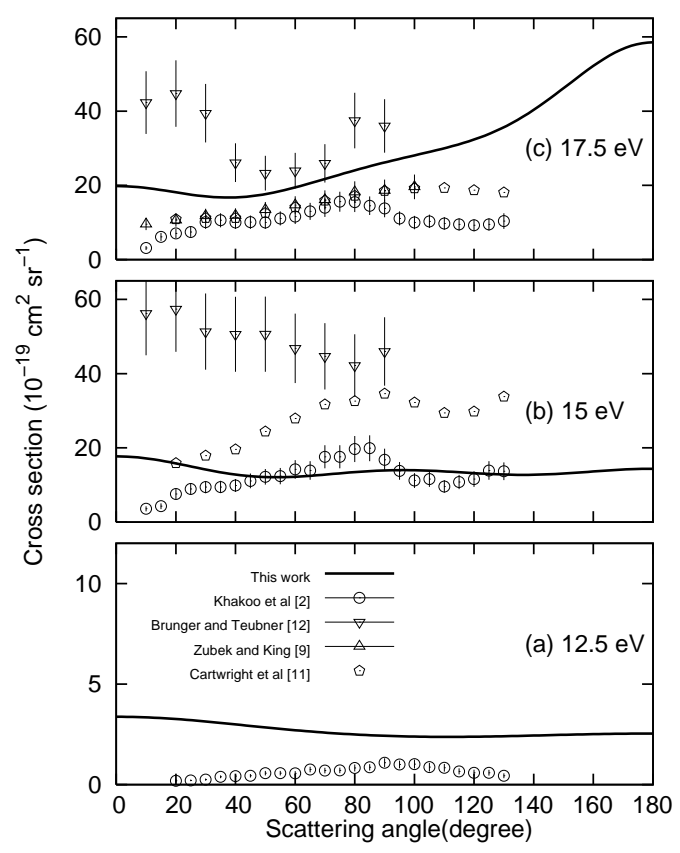

FIG. 11: Differential cross sections for electron impact excitation from the $\mathrm{N}_{2} X^{1} \Sigma_{g}^{+}$state to the $C^{3} \Pi_{u}$ state. Panel (a): electron impact energy of $12.5 \mathrm{eV}$, (b):15 eV and (c): $17.5 \mathrm{eV}$. The experimental DCSs of Zubek and King [9] are added. Other details are the same as in Fig,4, 
TABLE I: Division of the orbital set in each symmetry.

\begin{tabular}{lrrrrrrrr}
\hline \hline Symmetry & $A_{g}$ & $B_{2 u}$ & $B_{3 u}$ & $B_{1 g}$ & $B_{1 u}$ & $B_{3 g}$ & $B_{2 g}$ & $A_{u}$ \\
\hline Valence & $1-3 a_{g}$ & $1 b_{2 u}$ & $1 b_{3 u}$ & & $1-3 b_{1 u}$ & $1 b_{3 g}$ & $1 b_{2 g}$ & \\
Extra virtual & $4 a_{g}$ & $2 b_{2 u}$ & $2 b_{3 u}$ & $1 b_{1 g}$ & $4 b_{1 u}$ & $2 b_{3 g}$ & $2 b_{2 g}$ & $1 a_{u}$ \\
Continuum & $5-39 a_{g}$ & $3-35 b_{2 u}$ & $3-35 b_{3 u}$ & $2-17 b_{1 g}$ & $5-37 b_{1 u}$ & $3-18 b_{3 g}$ & $3-18 b_{2 g}$ & $2-17 a_{u}$ \\
\hline \hline
\end{tabular}


TABLE II: Comparison of the vertical excitation energies. The present results are shown with the previous works of Gillan et al.[21], multi reference coupled-cluster (MRCC) results of Ben-Shlomo and Kaldor [33] as well as experimental values quoted in Ben-Shlomo and Kaldor [33]. The unit of energy is eV.

\begin{tabular}{lrrrr}
\hline \hline State & This work & Previous R-matrix & MRCC & Experimental values \\
\hline$X^{1} \Sigma_{g}^{+}$ & 0.00 & 0.00 & 0.00 & 0.00 \\
$A^{3} \Sigma_{u}^{+}$ & 7.89 & 7.63 & 7.56 & 7.75 \\
$B^{3} \Pi_{g}$ & 8.54 & 8.54 & 8.05 & 8.04 \\
$W^{3} \Delta_{u}$ & 9.38 & 9.11 & 8.93 & 8.88 \\
$a^{1} \Pi_{g}$ & 9.85 & 9.89 & 9.27 & 9.31 \\
$B^{\prime 3} \Sigma_{u}^{-}$ & 10.06 & 9.83 & 9.86 & 9.67 \\
$a^{\prime 1} \Sigma_{u}^{-}$ & 10.69 & 10.41 & 10.09 & 9.92 \\
$w^{1} \Delta_{u}$ & 11.01 & 10.74 & 10.54 & 10.27 \\
$C^{3} \Pi_{u}$ & 11.64 & & 11.19 & 11.19 \\
\hline \hline
\end{tabular}

